\title{
Better anticoagulation control improves survival after valve replacement
}

\author{
Eric G. Butchart, FRCS, FESC \\ Nicola Payne, MPhil ${ }^{b}$ \\ Hui-Hua Li, MD ${ }^{\mathrm{b}}$ \\ Keith Buchan, FRCS ${ }^{a}$ \\ Kayapanda Mandana, $\mathrm{MD}^{\mathrm{a}}$ \\ Gary L. Grunkemeier, PhD ${ }^{\text {b }}$
}

From the Department of Cardiothoracic Surgery, ${ }^{a}$ University Hospital of Wales, Cardiff, United Kingdom, and the Medical Data Research Centre, ${ }^{\mathrm{b}}$ Providence Health System, Portland, Ore.

Funding for this study was provided by Medtronic, Inc, Minneapolis, Minn.

Read at the Twenty-seventh Annual Meeting of The Western Thoracic Surgical Association, San Diego, Calif, June 20-23, 2001.

Received for publication June 28, 2001; accepted for publication Sept 26, 2001.

Address for reprints: Eric G. Butchart, FRCS, FESC, Department of Cardiothoracic Surgery, University Hospital of Wales, Heath Park, Cardiff CF14 4XN, United Kingdom (E-mail: egbutchart@ aol.com).

J Thorac Cardiovasc Surg 2002;123:715-23

Copyright (C) 2002 by The American Association for Thoracic Surgery

0022-5223/2002 $\$ 35.00+0 \quad \mathbf{1 2 / 6 / 1 2 1 1 6 2}$

doi:10.1067/mtc.2002.121162

Objective: We sought to assess the effect of anticoagulation control on long-term survival after valve replacement with the Medtronic Hall valve (Medtronic, Inc, Minneapolis, Minn).

Methods: Prospective follow-up data, including 82,297 international normalized ratios, were collected for 1476 patients undergoing single valve replacement with the Medtronic Hall valve between 1979 and 1994, with follow-up to the end of 1998. After excluding 204 patients who either died within 30 days or had fewer than 10 international normalized ratios recorded beyond 30 days, there were 10,203 patient years of follow-up for analysis. Anticoagulation variability was measured as the percentage of international normalized ratios outside a target range of 2.0 to 4.0 for each patient.

Results: Linearized rates for late death rose progressively with increasing deciles of anticoagulation variability for both aortic and mitral valve replacement $(2.7 \%$ and $3.3 \%$ per year, respectively, in deciles 1 and 2 up to $9.5 \%$ and $14.6 \%$ per year, respectively, in deciles $6-10 ; P<.001)$. Survival at 15 years after aortic valve replacement was $59 \%$ for low anticoagulation variability (deciles 1 and 2), 55\% for intermediate anticoagulation variability (decile 3), and $28 \%$ for high anticoagulation variability (deciles 4-10); survivals at 15 years after mitral valve replacement were $56 \%, 42 \%$, and $24 \%$, respectively $(P<.001$ between low-intermediate anticoagulation variability and high anticoagulation variability for both aortic and mitral valve replacement). On multivariate analysis, significant predictors of reduced survival were anticoagulation variability per $20 \%$ increase (hazard ratio, 1.8), diabetes (hazard ratio, 1.6), decade of age (hazard ratio, 1.6), concomitant coronary artery bypass grafting (hazard ratio, 1.5), male sex (hazard ratio, 1.4), hypertension (hazard ratio, 1.4), New York Heart Association class III or IV (hazard ratio, 1.3), and non-sinus rhythm (hazard ratio, 1.2). Patients with low anticoagulation variability who were in sinus rhythm and did not have diabetes, coronary bypass grafting, or hypertension had survivals equal to those of the age- and sex-matched general population at 15 years. The incidence of valve-related deaths was significantly higher with high anticoagulation variability compared with the incidence with low-intermediate anticoagulation variability for both aortic $(1.4 \%$ vs $0.5 \%$ per year, $P<.001)$ and mitral valve replacement $(1.5 \%$ vs $0.5 \%$ per year, $P<.001)$. By means of univariate analysis, high anticoagulation variability was significantly associated with New York Heart Association class III or IV at 5 years postoperatively $(P<.001)$ and with age of greater than 60 years at the time of the operation $(P=.002)$.

Conclusions: High anticoagulation variability is the most important independent predictor of reduced survival after valve replacement with a mechanical valve. Better anticoagulation control should improve survival. 


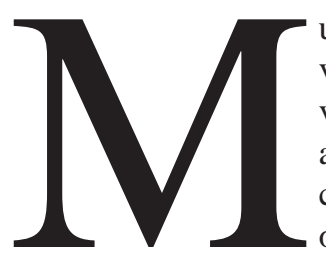

uch of the debate about prosthetic valve choice between mechanical valves and bioprostheses revolves around anticoagulation and its perceived disadvantages. ${ }^{1-3}$ Although oral anticoagulation with coumarin derivatives has been used therapeutically for 60 years, ${ }^{4}$ and for patients with prosthetic heart valves in particular for 40 years, it is only in the last 15 years that anticoagulation has been placed on a safer and more scientific basis. Progress toward safer anticoagulation began with the introduction of standardization of anticoagulation measurement with the international normalized ratio (INR) in the mid-1980s. ${ }^{5}$

Oral anticoagulation has always been monitored with the prothrombin time test, in which a thromboplastin reagent containing tissue factor is added to the patient's blood, and the time taken for a clot to form is compared with that in normal blood. Thromboplastin reagents are biologic products, typically made from rabbit brain, that vary in their content of tissue factor from one commercial product to another. Although different thromboplastin reagents yield very similar results with normal blood, they may produce widely differing prothrombin times with anticoagulated blood. Before the introduction of the INR, the intensity of anticoagulation was expressed in simple unstandardized terms either as the prothrombin time or as a prothrombin ratio in comparison with normal blood. In some European countries the anticoagulation effect was expressed as a percentage of normal prothrombin activity remaining (eg, Thrombotest and Hepato-Quick).

Because of the uncertainty about the true intensity of anticoagulation, it was clear that some patients were being anticoagulated at unnecessarily high intensities, particularly in North America, and thus exposed to a greater risk of bleeding. ${ }^{6}$ The introduction of the INR system depended on calibration of thromboplastin reagents. Each commercial reagent was accorded an international sensitivity index, allowing correction of the raw prothrombin ratio to the INR by using a simple nomogram. ${ }^{5}$ Although the INR system was adopted widely in Europe from the mid-1980s onward, there was initial reluctance to use the new system in North America, and it did not become widely used in the United States until the late 1990s. ${ }^{7}$

The ability to express anticoagulation intensity in standardized terms allowed better international communication on this subject and led to progress in defining the optimum INR range for many conditions and for patients with prosthetic heart valves. ${ }^{8}$ Although not fully worked out for all types of mechanical valves and all thrombotic risk factors because of a lack of data, there is now widespread acceptance of the principle that the target INR range should be both prosthesis specific and patient specific. ${ }^{9}$ In general there has been a lowering of recommended INRs for low- thrombogenicity prostheses in recent years, with an associated reduction in the risk of serious bleeding. ${ }^{10}$

With standardization of measurement and established target ranges for some individual types of prostheses, the only remaining challenge is to improve anticoagulation control. Some progress has been made in this area with the demonstration, in several studies, that patient self-management with home prothrombin measurement devices results in a greater percentage of time within the target INR range. ${ }^{11,12}$ Two studies have also shown a reduction in both thromboembolic and bleeding events with patient self-management. ${ }^{13,14}$ These studies and others that have shown the effect of good conventional anticoagulation control in preventing adverse events emphasize the importance of this aspect of anticoagulation management. ${ }^{15}$

Although the prevention of thromboembolism and serious bleeding are essential goals in themselves, the effect of anticoagulation control on long-term survival in patients with prosthetic heart valves has not previously been addressed. We therefore used our 20-year experience with the Medtronic Hall valve (Medtronic, Inc, Minneapolis, Minn) to investigate this aspect. We have previously used this database to define the optimum INR for the Medtronic Hall valve, ${ }^{16}$ to demonstrate the importance of stroke risk factors in determining susceptibility to thromboembolic events after valve replacement with this prosthesis, ${ }^{17,18}$ and to document the good long-term performance of the Medtronic Hall valve. ${ }^{19}$ In patients with single valve replacement, 11,174 patient-years of follow-up and 82,297 individual INR observations were available for analysis.

\section{Methods}

The INR system was introduced in the United Kingdom in the mid-1980s. Before this, anticoagulation data in this study were collected as the British corrected ratio, which numerically gave values almost identical to the INR in the therapeutic range. Hence British corrected ratio and INR data were amalgamated for the purpose of this analysis, and all data are described in terms of the INR.

The follow-up methods and the anticoagulation protocols used in our 20-year Medtronic Hall valve study have already been described. ${ }^{19}$ Between 1979 and the mid-1980s, all patients were given a target INR of 2.0 to 3.0. After general recommendations from European and British hematologists in the mid-1980s that all patients with prosthetic heart valves (irrespective of type) should be given a target INR range of 3.0 to 4.5 , this higher range was implemented by most of the anticoagulant clinics that our patients attended. In practice, the new higher recommendations were introduced cautiously, and although most patients were managed in the range of 3.0 to 4.0 , there was considerable variability in achieved INRs among the 27 different anticoagulation clinics that the patients attended, with median INR values varying from 2.2 to $3.9 .{ }^{20}$

Interim analysis of the effect of the new recommendations on patients in the Medtronic Hall valve study, in terms of the incidence of thromboembolism and bleeding, led to the definition of ideal target ranges for patients with this prosthesis, ${ }^{16}$ and from 1990 onward, patients with aortic valve replacement (AVR) were 
advised to maintain their INR between 2.0 and 3.0. Patients with mitral valve replacement (MVR) and all patients in atrial fibrillation were prescribed a range of 3.0 to 4.0. INRs were measured at 4- to 6-week intervals but more frequently if very low or very high INRs were recorded $(<1.7$ or $>5.0)$. None of the patients in this study managed their own anticoagulation.

Thus, throughout the study, all patients were managed within the INR range of 2.0 to 4.0. For the purposes of this analysis, variability of anticoagulation control (ACV) for each patient was expressed as the percentage of INR values outside the 2.0 to 4.0 range. ACV data, which ranged from $0 \%$ to $100 \%$, were grouped into deciles and then divided into 3 approximately equal-sized groups defined as low (0\%-19.9\% ACV), intermediate (20\%$29.9 \% \mathrm{ACV}$ ), and high ( $\geq 30 \%$ ) ACV. Only INR values, events, and deaths after the first 30 days were analyzed because the purpose of the investigation was to assess the effect of long-term anticoagulation control. Patients were excluded from the analysis if they had fewer than 10 documented INR values. The average number of INR values per patient was 64 (range, 10-331).

The survival of individual patients was examined in relation to $\mathrm{ACV}$ and in relation to the patient risk factors already identified as predicting reduced survival: diabetes, hypertension, concomitant coronary artery bypass grafting, and non-sinus rhythm. ${ }^{19}$ Mortality statistics for England and Wales from 1993 to 1995 (Series DH1, number 28, Office for National Statistics, London, United Kingdom) were used for survival comparisons with the age- and sexmatched general population.

All analyses were performed with the SPLUS software package, version 2000 (Insightful, Inc, Seattle, Wash). Linearized rates (LRs) for death by decile of ACV were graphically displayed by circles proportional in size to the number of follow-up years and color coded according to low (light gray), intermediate (white), and high (dark gray) ACV by using the criteria defined above. Patient survival curves were constructed with the actuarial method of Kaplan and Meier. ${ }^{21}$ Survivals in ACV and risk factor groups were compared by using the log-rank method. The Cox proportional hazard model was used to assess the relationship between $\mathrm{ACV}$, risk factors, thromboembolism, and major bleeding events after valve replacement and late survival. ${ }^{22}$ Thromboembolism and major bleeding events were treated as time-dependent covariates in the Cox regression analysis. LRs were compared by using the likelihood ratio test. ${ }^{23}$ Associations between ACV and New York Heart Association (NYHA) class at 5 years postoperatively and age were tested by using the $\chi^{2}$ test.

\section{Results}

Patient characteristics and clinical variables for single aortic and mitral procedures $(\mathrm{n}=1532)$ are summarized in Table 1. INR data were collected on $1476(96 \%)$ patients. Of these, 204 patients were excluded because they had 30 or fewer days of follow-up (early deaths) or less than 10 INR values. Of the remaining 1272 patients $(10,203$ total patientyears; mean, 8.0 years; SD, 4.2 years; maximum, 18 years), 625 had AVRs (4684 total patient-years; mean, 7.5 years; SD, 3.9 years; maximum, 18 years), and 647 had MVRs (5519 total patient-years; mean, 8.5 years, SD, 4.5 years; maximum, 18 years). Late mortality was $3.7 \%$ per year for AVR and $4.9 \%$ per year for MVR.
TABLE 1. Clinical variables by valve position

\begin{tabular}{|c|c|c|}
\hline & Aortic & Mitral \\
\hline No. of replacements & 625 & 647 \\
\hline Male $(\%)$ & 69 & 27 \\
\hline \multicolumn{3}{|l|}{ Age (years) } \\
\hline Mean $\pm S D$ & $60 \pm 11$ & $57 \pm 10$ \\
\hline Range & $20-89$ & $13-79$ \\
\hline \multicolumn{3}{|l|}{ NYHA (\%) } \\
\hline $1-2$ & 61 & 43 \\
\hline $3-4$ & 39 & 57 \\
\hline \multicolumn{3}{|l|}{ Previous symptoms (\%) } \\
\hline Angina pectoris & 44 & 16 \\
\hline Orthopnea & 38 & 66 \\
\hline Myocardial infarction & 8 & 8 \\
\hline Heart failure & 29 & 47 \\
\hline Atrial fibrillation/flutter & 10 & 69 \\
\hline Thromboembolism & 6 & 17 \\
\hline \multicolumn{3}{|l|}{ Concomitant disease $(\%)$} \\
\hline Hypertension & 21 & 12 \\
\hline Diabetes mellitus & 5 & 6 \\
\hline Emergency operation (\%) & 9 & 5 \\
\hline \multicolumn{3}{|l|}{ Previous valve operation (\%) } \\
\hline Valve repair/valvotomy & 2 & 28 \\
\hline Valve replacement & 5 & 8 \\
\hline \multicolumn{3}{|l|}{ *Valve pathology $(\%)$} \\
\hline Rheumatic & 9 & 73 \\
\hline Endocarditis & 5 & 2 \\
\hline Congenital & 52 & 1 \\
\hline Ischemic & 0 & 4 \\
\hline Degenerative & 29 & 7 \\
\hline Calcific (\%) & 82 & 46 \\
\hline \multicolumn{3}{|l|}{ Operative diagnosis (\%) } \\
\hline Stenosis & 59 & 53 \\
\hline Regurgitation & 21 & 26 \\
\hline Mixed lesion & 20 & 21 \\
\hline
\end{tabular}

*The sum exceeds $100 \%$ because some valves have more than one pathology.

Of all the INR values collected, $75.5 \%$ were within the target range. Of the $24.5 \%$ of INR values outside the range, $12.0 \%$ were below 2.0, and $12.5 \%$ were above 4.0 . ACV ranged from $0 \%$ to $100 \%$, with an average of $27 \%$ (SD, $13 \%$ ) and a distribution that was slightly skewed to the right (Figure 1).

LRs for death rose with increasing deciles of ACV. For AVR, LRs ranged from $2.7 \%$ per year in deciles 1 and 2 $(0 \%-19.9 \% \mathrm{ACV})$ to $7.3 \%$ per year in decile $5(40 \%-49.9 \%$ $\mathrm{ACV})$ and $9.5 \%$ per year in deciles 6 to $10(50 \%-100 \%$ ACV, Figure 2). LRs for MVR were higher than those for AVR, ranging from $3.3 \%$ per year in deciles 1 and 2 to $10.3 \%$ per year in decile 5 and $14.6 \%$ per year in deciles 6 to 10 (Figure 3). For both AVR and MVR, LRs for death in the low (light gray circles) and intermediate (white circles) ACV groups were similar but were significantly lower than in the high ACV group $(P<.001)$.

Survival at 5 years after AVR was $95 \%$ in the low ACV group and $93 \%$ in the intermediate ACV group; these results 


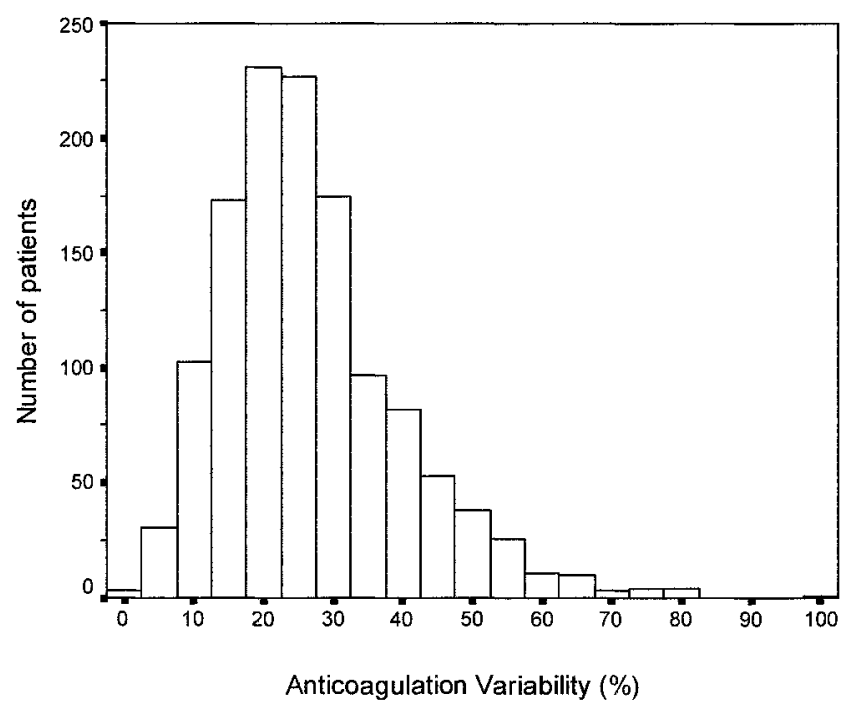

Figure 1. The distribution of ACV data in 1272 patients.

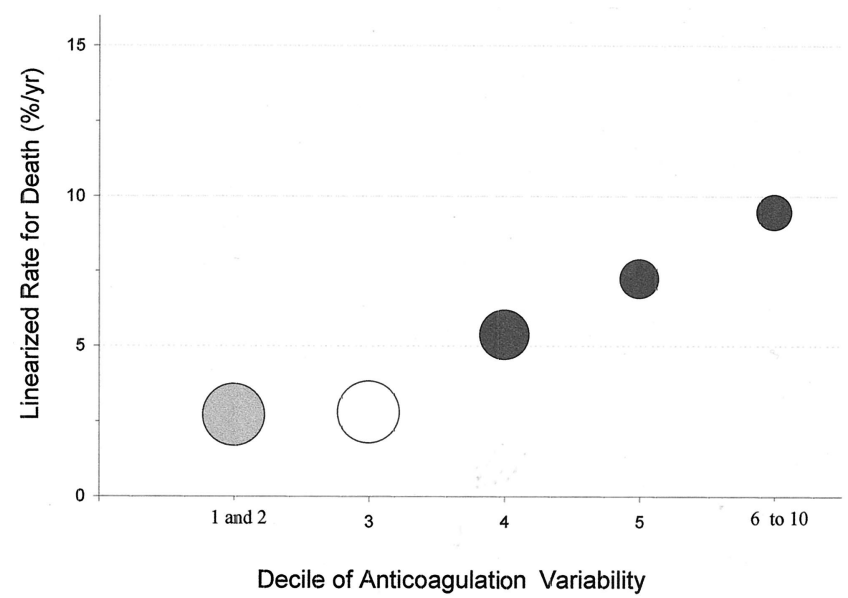

Figure 2. LRs for late death ( $>\mathbf{3 0}$ days) after AVR by decile of ACV. Decile 1 is $0 \%$ to $9.9 \%$ ACV, decile 2 is $10 \%$ to $19.9 \%$ ACV, and so on up to decile 10 (90\%-100\% ACV). Deciles 1 and 2 have been combined to give one circle, which represents low variability. Deciles $\mathbf{6}$ to $\mathbf{1 0}$ have been combined to achieve a minimum of 200 patient-years. The area of each circle is proportional to the number of patient-years of follow-up in that decile. The circles are color coded according to low (0\%-19.9\% ACV; light gray), intermediate (20\%-29.9\% ACV; white), and high ( $\geq 30 \% \mathrm{ACV}$; dark gray) variability.

were not significantly different from those of the UK population. In the high ACV group the survival was significantly lower $(77 \%, P<.001$ compared with the survival of the low ACV group). Survival at 15 years was similar for the low $(59 \%)$ and intermediate $(55 \%)$ ACV groups but significantly reduced in the high ACV group $(28 \%, P<$ .001; Figure 4). Results for MVR showed a similar pattern, but survivals were lower than those for AVR; survival at 5

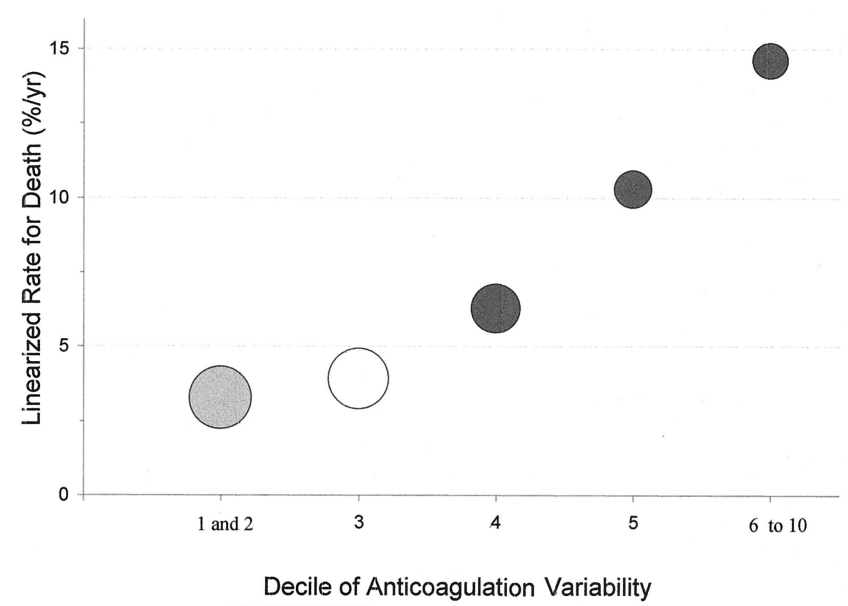

Figure 3. LRs for late death after MVR by decile of ACV. For details, see legend to Figure 2.

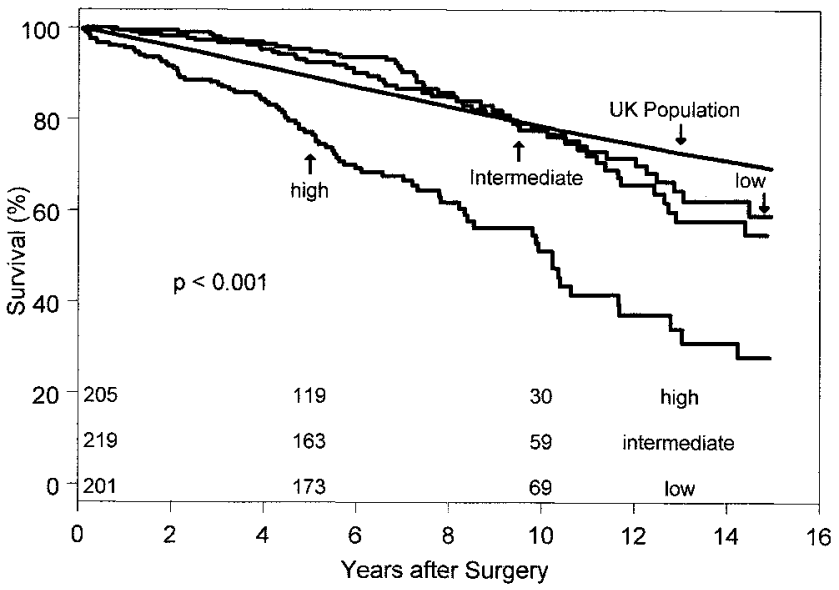

Figure 4. Late survival ( $>30$ days) by ACV for AVR. ACV is grouped according to low (0\%-19.9\% ACV), intermediate (20\%-29.9\% ACV), and high ( $\geq 30 \% \mathrm{ACV}$ ) variability. The numbers above the axis represent the number of patients at risk at 0,5 , and 10 years, respectively, within each ACV group.

years was $92 \%, 90 \%$, and $67 \%$ for the low, intermediate, and high ACV groups, respectively, and survival at 15 years was 56\%, 42\%, and 24\%, respectively (Figure 5).

Survivals for patients with no risk factors and low or intermediate ACV were not significantly different from those of the UK population. With increasing numbers of risk factors, survivals declined. At 15 years, survival was $45 \%$, $21 \%$, and $13 \%$ for groups with 1,2 , and 3 or more risk factors, respectively (Figure 6).

The incidence of valve-related deaths (including stroke, thrombosis, major bleeding, and prosthetic valve endocarditis) was significantly higher in the high ACV group compared with that in the low-intermediate ACV group both for AVR (LRs $1.4 \%$ vs $0.5 \%$ per year, $P<.001$ ) and MVR 
$(1.5 \%$ vs $0.5 \%$ per year, $P<.001)$. For AVR, there was a significantly higher incidence of strokes in the high ACV group compared with that in the low-intermediate ACV group $(0.7 \%$ vs $0.1 \%$ per year, $P<.001)$ and a trend toward a higher incidence of major bleeding and prosthetic valve endocarditis. For MVR, the linearized rate of prosthetic valve endocarditis was significantly higher in the high ACV group ( $0.6 \%$ vs $0.07 \%$ per year, $P<.001)$, and there was a trend toward a higher incidence of stroke, thrombosis, and major bleeding in this group (Table 2).

By means of univariate Cox analysis, significant predictors of reduced survival were traditional risk factors and ACV $(P<.001)$, major bleeding events $(P=.007)$, thromboembolism $(P=.038)$, and both thromboembolism and major bleeding events $(P=.001)$. By means of multivariate analysis, significant predictors were ACV per $20 \%$ increase (hazard ratio [HR], 1.8), diabetes (HR, 1.6), decade of age (HR, 1.6), concomitant coronary artery bypass grafting (HR, 1.5), male sex (HR, 1.4), hypertension (HR, 1.4), thromboembolism and major bleeding events (HR, 1.3), valve regurgitation (HR, 1.3), NYHA class III or IV (HR, 1.3), non-sinus rhythm (HR, 1.2), and small valve size (HR, 1.1; Table 3). Valve position was not a significant factor in the model.

By means of univariate analysis, high ACV was significantly associated with higher NYHA class (III and IV) at 5 years postoperatively and with older age. In the low-intermediate ACV group, $6 \%$ were in NYHA class III or IV, and $44 \%$ were above the age of 60 years compared with $12 \%$ and $58 \%$ in the high ACV group, respectively $(P<.001$ for NYHA and .002 for age).

\section{Discussion}

Although the importance of good-quality anticoagulation control in reducing the risk of thromboembolism and bleeding is generally acknowledged, the current analysis demonstrates for the first time the major effect of anticoagulation control on long-term survival. High variability in INR was the strongest independent predictor of reduced survival, and there was a $32 \%$ difference in survival at 15 years between patients with low and high ACV. When other risk factors for reduced survival were taken into account and survival was related to numbers of risk factors (including high $\mathrm{ACV}$ ), there was a $55 \%$ difference in survival at 15 years between patients with no risk factors and those with 3 or more risk factors. The survival of patients with no risk factors was no different from that of the age- and sex-matched general population.

LRs of valve-related death were significantly higher in patients with high ACV for both AVR and MVR. Not only were there more deaths from stroke and major bleeding events in patients with high ACV, as expected, but among patients undergoing MVR, there was a 9-fold increase in prosthetic endocarditis, which was highly significant $(P<$

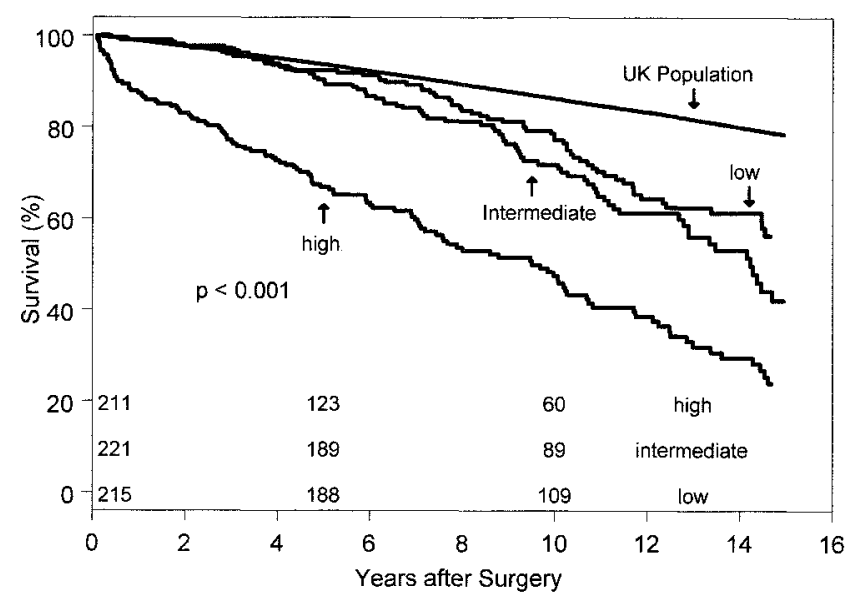

Figure 5. Late survival by ACV for MVR. For details, see legend to Figure 4.

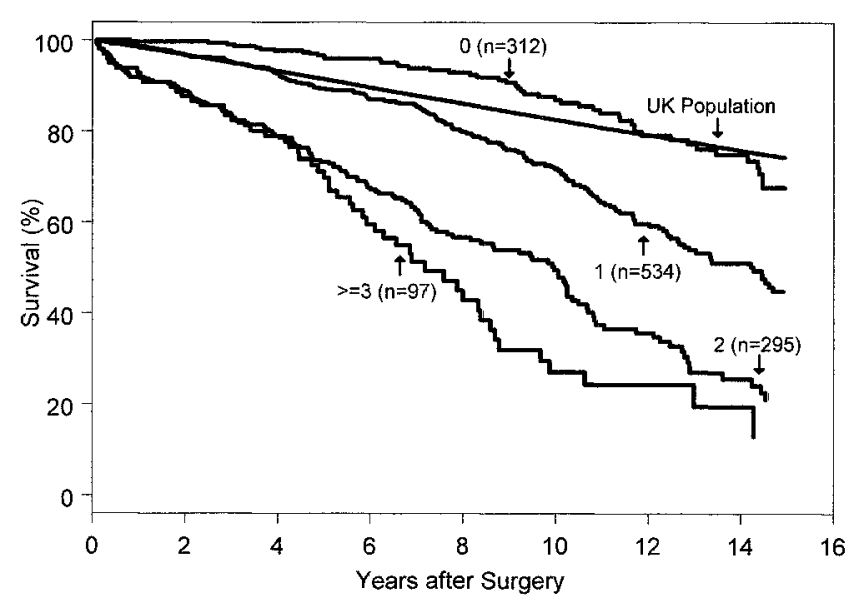

Figure 6. Late survival by number of risk factors (both valve positions). Risk factors are diabetes, hypertension, concomitant coronary artery bypass grafting, non-sinus rhythm, and high ACV ( $\geq 30 \%$ ACV).

.001). There are 3 possible explanations for this interesting and unexpected finding. First, periods of low INR could result in subclinical deposition of thrombus on the sewing ring of the prosthesis, providing a culture medium for any circulating bacteria. Second, patients who are poorly compliant with anticoagulation may be poorly compliant with endocarditis prophylaxis and dental hygiene also. Third, patients whose high ACV is due to frequent prescription of antibiotics that interact with warfarin would clearly be exposed to more frequent infections. The most common cause of frequent antibiotic prescriptions is lower respiratory tract infection, and this would be in keeping with a strong association with prosthetic endocarditis in the mitral position. Thus, it is possible that the relationship between high ACV and prosthetic endocarditis is either causative or simply an 
TABLE 2. Valve-related deaths by ACV grouped

\begin{tabular}{lcccc}
\hline & \multicolumn{2}{c}{ Aortic } & \multicolumn{2}{c}{ Mitral } \\
\cline { 2 - 4 } & $\begin{array}{c}\text { Low/medium ACV } \mathbf{n} \\
(\% / \mathbf{y r})\end{array}$ & $\begin{array}{c}\text { High ACV } \\
\mathbf{n}(\% / \mathbf{y r})\end{array}$ & $\begin{array}{c}\text { Low/medium ACV } \mathbf{n} \\
(\% / \mathbf{r r})\end{array}$ \\
\hline Patients & 420 & 205 & 436 & 211 \\
Patient-years & 3454 & 1230 & 4106 & 1413 \\
Stroke & $5(0.1)$ & $8(0.7)^{* * *}$ & $8(0.2)$ & $5(0.4)$ \\
Thrombosis & $0(0)$ & $0(0)$ & $1(0.02)$ & $9(0.2)$ \\
Major bleeding & $8(0.2)$ & $6(0.5)$ & $3(0.07)$ & $7(0.5)$ \\
PVE & $3(0.09)$ & $3(0.2)$ & $21(0.5)$ & $8(0.6)^{* * *}$ \\
Total & $16(0.5)$ & $17(1.4)^{* * *}$ & $21(1.5)^{* * *}$ \\
\hline
\end{tabular}

$A C V$, Anticoagulant variability; $P V E$, prosthetic valve endocarditis.

***P $P$ <.001.

TABLE 3. Cox regression risk factors for late survival over a 20 -year period

\begin{tabular}{lccr}
\hline & Coefficient & P value & \\
\hline ACV (per 20\% increase) & 0.595 & .001 & 1.813 \\
Diabetes & 0.497 & .007 & 1.644 \\
Decade of age & 0.489 & .001 & 1.630 \\
Concomitant CABG & 0.413 & .002 & 1.511 \\
Male sex & 0.371 & .001 & 1.448 \\
Hypertension & 0.361 & .010 & 1.434 \\
TE/BE & 0.281 & .021 & 1.324 \\
Valve regurgitation & 0.252 & .016 & 1.287 \\
NYHA class III or IV & 0.245 & .020 & 1.277 \\
Non-sinus rhythm & 0.216 & .087 & 1.241 \\
Small valve size & 0.073 & .001 & 1.075 \\
\hline
\end{tabular}

$\overline{A C V}$, Anticoagulant variability; $T E$, thromboembolism; $B E$, major bleed; $H R$, hazard ratio.

association caused by other factors. Further investigation of this unexpected relationship is required.

Overall, the findings in this study have implications for the interpretation of prosthetic valve data and for the anticoagulation management of all patients with prosthetic heart valves.

\section{Prosthetic Heart Valve Performance}

Traditionally, prosthetic heart valves, whether mechanical or biologic, have been compared in terms of patient survival and the incidence of adverse events. ${ }^{1}$ This study confirms the findings of others that patient factors exert a major influence on survival ${ }^{24-28}$ but also shows that these factors are additive in their effect and that ACV (never measured in most studies) is the most important independent factor.

Determining the effect of the prosthesis itself on survival in any study is fraught with difficulties because even comparison with age- and sex-matched general population data from the same country has limitations. On the one hand, there may be selection bias if only patients with good general performance status and no significant comorbidities are chosen for surgical treatment. On the other hand, in reality patients undergoing heart valve replacement tend to have a higher prevalence of risk factors that adversely affect survival (atrial fibrillation, left ventricular hypertrophy, and coronary artery disease) than the general population.

In this study patients who had no significant risk factors for reduced survival and low ACV had a 15-year survival identical to that of the general population. Although open to the criticism that the general population would also have some of these risk factors that were not excluded, it is likely that the prevalence of these risk factors in the general population would be much less and that today many would be treated effectively (eg, atrial fibrillation, hypertension, and coronary artery disease), thus reducing their effect on survival data. Although admittedly imperfect, comparison of survival with that of the general population, having excluded significant nonprosthetic risk factors, provides a better means of assessing prosthetic performance than unstratified survival data and may allow more meaningful comparison of one prosthesis with another.

The skeptics and proponents of bioprosthetic valves may say how much better it would be to avoid anticoagulation altogether because poorly managed anticoagulation control has such an adverse effect on survival. However, structural valve deterioration is a major determinant of reduced survival in patients with bioprostheses, ${ }^{2}$ and it could be argued 
that efforts to improve anticoagulation control can only result in an even greater overall survival advantage with mechanical valves than has already been demonstrated by the only randomized trial of mechanical valves versus bioprostheses to report 15-year results. ${ }^{2}$ Despite the use of first-generation tilting disc valves and a high incidence of bleeding events, probably associated with unstandardized anticoagulation measurement, this randomized trial was still able to demonstrate a survival advantage at 15 years in patients with mechanical aortic valves. A randomized trial carried out now, using the least thrombogenic mechanical valves and high-quality anticoagulation management, would be likely to show an even more marked survival advantage.

\section{Improving Anticoagulation Control}

Because anticoagulation control has such a dramatic influence on survival after valve replacement, it is essential that further efforts are made to improve control and to identify the causes of high ACV. The finding in this study that high variability in INR is significantly associated with higher NYHA class may be due to the fact that most patients with impaired performance status are taking multiple medications, raising the risk of warfarin interaction and INR instability. Functionally compromised patients may also be less compliant with medication and less compliant in attending anticoagulation clinics. High INR variability was also associated with older age. Older patients tend to have more comorbidities and consequently take a greater number of medications. They may also be more forgetful in taking their warfarin regularly. Studies of bleeding risk in patients taking anticoagulants have demonstrated the importance of multiple comorbidities in increasing the risk, ${ }^{29}$ and it is likely that this increased risk is mediated through high INR variability.

Other possible causes of high ACV in this study will be the subject of further analysis. We have already documented considerable variation in achieved INR among groups of patients attending 27 different anticoagulation clinics (median INR ranged from 2.2-3.9). ${ }^{20}$ Anticoagulation intensity that is maintained close to either end of the INR target range is more likely to go outside the range, and this may be an important factor. Test frequency has also been shown to have an effect on INR variability, with more frequent testing increasing the time in target range. ${ }^{5,12}$ Although long-acting coumarin derivatives, such as phenprocoumon (used in some European countries), are associated with more stable INR control, one study of 6814 patients showed that the incidence of bleeding complications was higher, possibly because a high INR will take longer to return to the target range. ${ }^{30}$

A study of compliance with anticoagulation carried out in the United States showed that younger, male patients who had not experienced any thromboembolic events were more likely to forego INR testing. On univariate analysis, other factors associated with poor compliance were nonwhite ethnic origin (odds ratio [OR], 6.4), working full time (OR, 5.6), and lack of health insurance (OR, 5.6). On multiple logistic regression, the latter 2 factors were not independently predictive, but nonwhite race remained marginally significant $(P=.06)$. Compliance was not affected by income, marital status, or education. ${ }^{31}$

Another recent American study comparing "usual medical care" with that in an anticoagulation clinic run by a clinical pharmacist showed that the latter treatment strategy improved anticoagulation control, reduced bleeding and thromboembolic event rates, and saved over $\$ 160,000$ per 100 patients annually in reduced hospitalizations and emergency department visits. ${ }^{32}$ Several studies have also shown the value of patient self-management in conjunction with home prothrombin testing in improving the percentage of time spent within the prescribed INR range. ${ }^{11-14,33}$ It is likely that the use of self-management will increase rapidly in those countries in which the portable measurement devices are now readily obtainable. In the future, it can be anticipated that most patients with prosthetic heart valves will manage their own anticoagulation with the same facility that persons with diabetes manage their insulin requirements and that self-management will become the norm, allowing patients to lead fuller, safer lives and to travel without restriction. Compliance is also likely to be improved if patients do not need to take time off work to attend an anticoagulation clinic or pay for their INR to be measured.

Good anticoagulation control also involves patient education, and most studies of self-management have emphasized this aspect. A recent randomized, multicenter trial evaluated a structured teaching and self-management program for anticoagulated patients in comparison with conventional anticoagulation management by family physicians and found not only more INR values in the target range but also higher patient satisfaction scores on a quality-of-life questionnaire. ${ }^{33}$ Patients need a basic understanding of anticoagulation and, if they choose, training in the use of a home prothrombin measurement device and warfarin dosage adjustment. They also need dietary advice about the antagonistic effects of foods containing large quantities of vitamin $\mathrm{K}^{34}$ and education about the effect on the INR of drug interactions with warfarin. ${ }^{35}$ Patients unable to undertake self-management may choose to delegate this responsibility to a close relative or may prefer to attend an anticoagulation clinic regularly. However, in this case choice of anticoagulation clinic is important. All anticoagulation clinics should audit their performance regularly according to agreed upon criteria, ${ }^{5}$ and these audit data should be made available to referring clinicians and patients. Despite high INR variability in some patients in this study, overall anti- 
coagulation management was reasonably good, with $75 \%$ of INR values within the target range. Other authors have reported as few as $50 \%$ or less of INR values in the target range, ${ }^{11,33,36}$ and the need for considerable improvement in the quality of anticoagulation management by individual physicians and by anticoagulation clinics has been emphasized. ${ }^{37}$

Whatever method is chosen, the potential for better anticoagulation control to improve survival in patients with prosthetic heart valves seems enormous and may outweigh the influence of other factors. Traditionally, surgeons seek technical solutions to improve long-term survival for their patients, but in the case of valve-replacement surgery, ensuring good long-term anticoagulation control is likely to have the greatest effect on survival. It is no longer sufficient simply to begin a patient on warfarin and hope for the best. Cardiac surgeons and physicians caring for patients with prosthetic valves have a major responsibility to ensure that their patients receive the best possible anticoagulation management.

\section{References}

1. Grunkemeier GL, Li HH, Naftel DC, Starr A, Rahimtoola SH. Longterm performance of heart valve prostheses. Curr Probl Cardiol. 2000;25:73-156.

2. Hammermeister K, Sethi GK, Henderson WG, Grover FL, Oprian C, Rahimtoola SH. Outcomes 15 years after valve replacement with a mechanical versus a bioprosthetic valve: final report of the Veterans Affairs randomised trial. J Am Coll Cardiol. 2000;36:1152-8.

3. Birkmeyer NJO, Birkmeyer JD, Tosteson ANA, Grunkemeier GL, Marrin CAS, O'Connor GT. Prosthetic valve type for patients undergoing aortic valve replacement: a decision analysis. Ann Thorac Surg. 2000;70:1946-52.

4. Link KP. The discovery of Dicumarol and its sequels. Circulation. 1959;19:97-107.

5. van den Besselaar AMHP, van der Meer FJM. Standardisation of oral anticoagulation measurement and management. In: Butchart EG, Bodnar $\mathrm{E}$, editors). Current issues in heart valve disease: thrombosis, embolism and bleeding. London: ICR Publishers; 1992. p. 277-92.

6. Hirsh J. Is the dose of warfarin prescribed by American physicians unnecessarily high? Arch Intern Med. 1987;147:769-71.

7. Ansell J, Hirsh J, Dalen J, Bussey H, Anderson D, Poller L, et al. Managing oral anticoagulant therapy. Chest. 2001;119:22S-38S.

8. Stein PD, Alpert JS, Bussey HI, Dalen J, Turpie AGG. Antithrombotic therapy in patients with mechanical and biological prosthetic heart valves. Chest. 2001;119:220S-7S.

9. Butchart EG. Prosthesis-specific and patient-specific anticoagulation. In: Butchart EG, Bodnar E, editors. Current issues in heart valve disease: thrombosis, embolism and bleeding. London: ICR Publishers; 1992. p. 293-317.

10. Butchart EG. Prosthetic heart valves. In: Verstraete M, Fuster V, Topol EJ, editors. Cardiovascular thrombosis. Philadelphia: Lippincott-Raven; 1998. p. 395-414.

11. Taborski U, Müller-Berghaus G. State-of-the-art patient self-management for control of oral anticoagulation. Semin Thromb Hemost. 1999;25:43-7.

12. Samsa GP, Matchar DB. Relationship between test frequency and outcomes of anticoagulation: a literature review and commentary with implications for the design of randomised trials of patient self-management. J Thromb Thrombolysis. 2000;9:283-92.

13. Bernardo A. Experience with patient self-management of oral anticoagulation. J Thromb Thrombolysis. 1996;2:321-5.
14. Horstkotte D, Piper C, Wiemer M. Optimal frequency of patient monitoring and intensity of oral anticoagulation therapy in valvular heart disease. J Thromb Thrombolysis. 1998;4:19-24.

15. Huber KC, Gersh BJ, Bailey KR, Schaff HV, Hodge DO, Cha RH, et al. Variability in anticoagulation control predicts thromboembolism after mechanical cardiac valve replacement: a 23 -year populationbased study. Mayo Clin Proc. 1997;72:1103-10.

16. Butchart EG, Lewis PA, Bethel JA, Breckenridge IM. Adjusting anticoagulation to prosthesis thrombogenicity and patient risk factors. Circulation. 1991;84(Suppl):III-61-9.

17. Butchart EG, Moreno de la Santa P, Rooney SJ, Lewis PA. The role of risk factors and trigger factors in cerebrovascular events after mitral valve replacement. J Card Surg. 1994;9(suppl):228-36.

18. Butchart EG, Moreno de la Santa P, Rooney SJ, Lewis PA. Arterial risk factors and cerebrovascular events following aortic valve replacement. J Heart Valve Dis. 1995;4:1-8.

19. Butchart EG, Li HH, Payne N, Buchan K, Grunkemeier GL. Twenty years' experience with the Medtronic Hall Valve. J Thorac Cardiovasc Surg. 2001;121:1090-100.

20. Butchart EG, Lewis PA, Kulatilake ENP, Breckenridge IM. Anticoagulation variability between centres: implications for prosthetic valve assessment. Eur J Cardiothorac Surg. 1988;2:72-81.

21. Kaplan EL, Meier P. Nonparametric estimation from incomplete observations. J Am Stat Assoc. 1958;53:457-81.

22. Cox DR. Regression methods and life tables. J R Stat Soc. 1972;34: 187-220.

23. Lee ET. Statistical methods for survival data analysis. Belmont, Calif: Lifetime Learning Publications; 1980.

24. Blackstone EH, Kirklin JW. Death and other time-related events after valve replacement. Circulation. 1985;72:753-67.

25. Lindblom D, Lindblom U, Qvist J, Lundström H. Long-term relative survival rates after heart valve replacement. $J$ Am Coll Cardiol. 1990;15:566-73.

26. McGiffin DC, O'Brien MF, Galbraith AJ, McLachlan GJ, Stafford EG, Gardner MA, et al. An analysis of risk factors for death and mode-specific death after aortic valve replacement with allograft, xenograft and mechanical valves. J Thorac Cardiovasc Surg. 1993; 106:895-911.

27. Petersteim DS, Cen Y-Y, Cheruvu S, Landolfo K, Bashore TM, Lowe $\mathrm{JE}$, et al. Long-term outcome after biologic versus mechanical valve replacement in 841 patients. J Thorac Cardiovasc Surg. 1999;117: 890-7.

28. Kvidal P, Bergström R, Hörte LG, Stahle E. Observed and relative survival after aortic valve replacement. J Am Coll Cardiol. 2000;35: 747-56.

29. Beyth RJ, Quinn LM, Landefeld CS. Prospective evaluation of an index for predicting the risk of major bleeding in outpatients treated with warfarin. Am J Med. 1998;105:91-9.

30. van der Meer FJM, Rosendaal FR, Vandenbroucke JP, Briet E. Bleeding complications in oral anticoagulant therapy. Arch Intern Med. 1993; 153:1557-62.

31. Arnsten JH, Gelfand JM, Singer DE. Determinants of compliance with anticoagulation: a case control study. Am J Med. 1997;103:11-7.

32. Chiquette E, Amato MG, Bussey HI. Comparison of an anticoagulation clinic with usual medical care. Arch Intern Med. 1998;158: 1644-7.

33. Sawicki PT. A structured teaching and self-management program for patients receiving oral anticoagulation. JAMA. 1999;281:145-50.

34. Booth SL, Charnley JM, Sadowski JA, Saltzman E, Bovill EG, Cushman M. Dietary vitamin K1 and stability of oral anticoagulation: proposal of a diet with constant vitamin K1 content. Thromb Haemost. 1997;77:504-9.

35. Wells PS, Holdbrook AM, Crowther NR, Hirsh J. Interactions of warfarin with drugs and food. Ann Intern Med. 1994;121:676-83.

36. Samsa GP, Matchar DB, Goldstein LB, Bonito AJ, Lux LJ, Witter $\mathrm{DM}$, et al. Quality of anticoagulation management among patients with atrial fibrillation. Arch Intern Med. 2000;160:967-73.

37. Ansell JE. The quality of anticoagulation management. Arch Intern Med. 2000;160:895-6. 


\section{Discussion}

Dr D. Craig Miller (Stanford, Calif). It is a pleasure to be asked to discuss an article by Eric Butchart. He is a widely respected cardiac surgeon who is also known as a meticulous, rigorous, and honest clinical investigator. You are also the father, Eric, of the concept of site-specific and valve type-specific anticoagulation. I think our patients have benefited from this advance.

Is it correct that the anticoagulation target values in your study complied with the global UK guidelines as set by the hematologists 2 to 4 ? You have previously shown conclusively for aortic mechanical valves (and particularly for the Medtronic-Hall valve) that an INR in the 2 to 3 range is fine, as is 3 to 4 for the mitral valves. What was your benchmark for adequacy of anticoagulation control in this study: the UK standards or your own personal standards?

Dr Butchart. You are quite right to say that during this very long period of the study, 20 years, we used slightly different INR ranges, but to look at ACV, we broadened the range to an INR of 2 to 4 . At one stage, we were managing all patients at an INR between 2 and 3, and then we followed national recommendations and went up to an INR of 3 to 4 or 4.5 .

Dr Miller. Okay. I have another question. If I saw one of your slides correctly, it indicated that there were 60 INRs per patient. Now, some of these persons have been followed for many, many years. Did that number actually mean 60 INR values per patient per year?

Dr Butchart. No, all together. That was an average of 64 per patient. The range was up to 300 per patient.

Dr Miller. Thank you. Was all this postoperative anticoagulation management performed in your UK anticoagulation clinics, which are run by the hematologists?

Dr Butchart. There is quite a lot of variation actually. Most are run by hematologists. Some are run by general physicians, and some are run by general practitioners.

Dr Miller. Which leads me to my next question. I was struck by the fact that there appears to be little or no home or patientcontrolled anticoagulation monitoring yet in Britain.

Dr Butchart. That's right. It is because the National Health Service can not afford it.

Dr Miller. Well, let's talk about that. Is it being done today, at least in your center in the UK?

Dr Butchart. No. We have not started it yet. We are considering starting a study looking at that, but the problem and the reason it has not been introduced in the UK is simply one of cost.
I did not have time to show the data, but there are at least 5 studies in the literature now that have shown significant benefit from use of home prothrombin measurements and patients managing their own anticoagulation.

Dr Miller. We know from the Scandinavian and German experiences that patient benefit and safety are provided by home anticoagulation control, but are there not also favorable cost implications?

Dr Butchart. Yes, I think there will be positive cost implications, with savings in hospital admissions and in patients making visits to the doctor to have their INRs measured.

Dr Miller. I am sorry to say that self-anticoagulation at home probably still is not widely used in the United States, although it is Food and Drug Administration approved. This is because many insurance companies seem to balk at paying for the machines and the disposable supplies. Therefore, we also may be a third-world country when it comes to this aspect of health care.

Looking at the HRs, the ones that had the biggest effect on survival were variability of anticoagulation and age, which I believe was 1.2 per decade. That seemingly low risk adds up if you talk about many decades. The other patient-related risk factors had only very small effects on survival. What I learned from you-and the data do stand on their own merits and the conclusions are well substantiated-is that how well you control the patient's postoperative anticoagulation is probably a whole heck of a lot more important than what type of mechanical valve you put in.

Dr Butchart. Yes. Almost certainly, that's right. Probably the single most important thing that we can do for our patients is to ensure that they have good-quality anticoagulation control.

Dr Miller. In the future, do you have any new drugs coming on the scene in Europe, for example new recombinant warfarin-type compounds or direct antithrombin inhibitors? Is there ever going to be an oral anticoagulant that is safer than warfarin? We forget that the warfarin and dicumerol family of drugs has now been around for over 45 years.

Dr Butchart. There may well be eventually, but I think we are a long way off from that.

Dr Miller. Still?

Dr Butchart. Yes.

Dr Miller. Very nicely presented, Eric. I think it is fair to say that most in the audience believe you. Thank you for being here with us.

Dr Butchart. Thank you very much, indeed. 